Research Paper

\title{
Association between Anaesthetic Technique and Oncological Outcomes after Colorectal Carcinoma Liver Metastasis Resection
}

\author{
Hao Gao ${ }^{1,2^{*}}$, Xiao-Yan Meng1 ${ }^{*}$, Hong-Qian Wang ${ }^{1 *}$, Feng-Feng Zhu ${ }^{4}$, Ai-Ling Guo ${ }^{1}$, Mei Zhu ${ }^{1}$, Jin-Chao
} Song ${ }^{\circledR}$, Wei-Feng $\mathrm{Yu}^{1,3}{ }^{\boxplus}$

1. Department of Anesthesiology, Eastern Hepatobiliary Surgery Hospital, the Second Military Medical University, 225 Changhai Road, Shanghai, China

2. Department of Anesthesiology, Shanghai Shuguang Hospital, Shanghai University of Traditional Chinese Medicine, 528 Zhangheng Road, Shanghai, China.

3. Department of Anesthesiology, Renji Hospital, School of Medicine, Shanghai Jiao Tong University, 160 Pudian Road, Shanghai, China.

4. Department of Hepatobiliary Surgery, the First Affiliated Hospital of the University of South China, 69 Chuanshan Road, Hunan, China

*Hao Gao, Xiao-Yan Meng and Hong-Qian Wang contributed equally to this work.

$\triangle$ Corresponding authors: Jin-Chao Song; sjch2013@163.com and Wei-Feng Yu; shawn@smmu.edu.cn

(C) Ivyspring International Publisher. This is an open access article distributed under the terms of the Creative Commons Attribution (CC BY-NC) license (https://creativecommons.org/licenses/by-nc/4.0/). See http://ivyspring.com/terms for full terms and conditions.

Received: 2018.06.20; Accepted: 2018.12.22; Published: 2019.01.24

\begin{abstract}
Background: Recently published studies suggest that the anaesthetic technique used during oncologic surgery can improve patient outcomes. Therefore, the authors evaluated the survival of patients with resected colorectal carcinoma liver metastases (CRCLMs) who received either EGA (general anaesthesia [GA] combined with epidural anaesthesia [EA]) or GA alone.

Methods: We conducted an ambispective cohort study including 225 post-surgical CRCLM patients between May 2007 and July 2012 and performed a follow-up investigation of survival in July 2017.

Results: The basic characteristics in the two groups were largely similar. The median (quartiles) recurrence interval for all patients was $10(2.5,23)$ months, and the median (quartiles) survival for CRCLM patients post-surgically was $37(30.5,51.5)$ months. Perioperative EA was associated with survival ( $P=0.039$, log-rank test), with an estimated hazard ratio of $0.737(95 \% \mathrm{Cl} 0.551-0.985)$ in the univariate analysis. Kaplan-Meier estimates of survival for GA and EGA suggested that GA might provide better outcomes than EGA $[P=0.028$, hazard ratio of $0.7328(95 \% \mathrm{Cl} 0.5433-0.9884)]$. Significant differences in anaesthesia techniques were found $(\mathrm{P}=0.048)$, with an adjusted estimated hazard ratio of 0.741 (95\% Cl $0.550-0.998)$ in the multivariate analysis. Subgroup analyses of patients in different age groups ( $<40, \geq 40$ but $<60$, and $\geq 60$ years old) suggested that no significant differences existed among all three subgroups.

Conclusions: Compared with EGA, GA may provide a better survival outcome for CRCLM patients. The benefits of anaesthetic techniques in oncological surgery are most likely related to certain cancer types.
\end{abstract}

\section{Introduction}

Colorectal carcinoma $(\mathrm{CRC})$ remains one of the most common malignancies in China and ranks as the third most prevalent cancer worldwide.[1,2] Approximately half of CRC patients develop liver metastases (LMs), and $90 \%$ will die from this cancer.[3,4] Surgical resection of colorectal carcinoma liver metastases (CRCLMs) has been proven to be the only potentially curative therapy with a 5-year survival rate of up to 50\%.[5-8] Many factors could affect the prognosis for overall survival, including microscopic status of the resected margin, number of metastases, a positive resection margin, extra-liver metastasis, degree of differentiation, depth of wall invasion, positive lymph node metastasis, and 
interval between the CRC operation and detection of CRCLMs.[9,10] In addition to these prognostic or predictive factors, the anaesthesia techniques applied in the procedure, which have profound implications for the prognosis of the procedure, are often neglected to a certain extent.

Epidural anaesthesia (EA) has been reported to preserve the perioperative immune response and to further affect cancer recurrence and metastasis in patients and in animals.[11] In addition, combined general-epidural anaesthesia (EGA) is frequently applied in major thoracic or abdominal surgery, which may provide stable haemodynamics and less medication or can even reduce cancer recurrence and prolong cancer-free survival in different kinds of procedures, although some studies have shown no significant improvement in survival compared to that with general anaesthesia (GA).[12-16] The mechanical basis underlying this phenomenon might be related to the effects of the anaesthetic technique or of specific anaesthetic drugs on immunology and cancer cell biology.[17] However, current publications often have limited sample sizes and other factor variations. Comprehensive studies are needed to provide further evidence that the combination of EA and GA might be related to improve overall survival.

There is no global consensus on the better choice of anaesthesia techniques related to operations for CRCLM after surgery, as studies in this field are scarce.[11] Withal, the choice of optimal anaesthesia techniques for complex operations and diseases has long been neglected. Previous studies and metaanalyses revealed that EGA might be associated with an improvement in the prognosis of patients with operable prostate cancer. Regarding the prognosis of colorectal cancer, no obvious improvement of EGA was detected compared to GA.[18] This result, although coming from the analysis of heterogeneous data, suggests that the applicability and priority of EGA or GA might be related to survival outcome. Thus, in considering the complexity of the disease and the benefits to patients, it is necessary to evaluate the priority of anaesthesia techniques for surgical resection of CRCLMs.

In this study, we compared the effects of EGA with those of GA on survival associated with CRCLM after surgery. We aimed to explore the advantages and disadvantages of EGA, compared to those of GA and based on this particular type of surgery, and to provide clinicians with further evidence for better decisions regarding the choice of anaesthesia techniques, via a large hospital-based ambispective cohort in China.

\section{Method}

\section{Patients Enrolled}

This ambispective cohort study was approved by the Ethics Committee of the Eastern Hepatobiliary Surgical Hospital of China (EHBHKY2012-002-028). Written informed consent was obtained from participants or their surrogates before enrolment. After the primary study design and hypothesis proposal, we reviewed the data of patients with CRCLMs after surgery between May 2007 and July 2012 from hospital medical records of the Eastern Hepatobiliary Surgery Hospital, Shanghai, China. Then, we conducted a follow-up investigation of treatment outcomes until July 2017. Patients were enrolled in this cohort if (1) they had been diagnosed with CRCLM after surgery; (2) the pathological classification of original colorectal cancer was moderately or highly differentiated tubular adenocarcinoma, Dukes' stages B-C; (3) the patients had received treatment by hepatic metastasis resection with R0 excision;[19] (4) the anaesthesia method was GA or EGA; (5) lesions were located in the liver with no lymphovascular invasion; and (6) there was no severe systemic disease and metastases in other organs before hepatic resection. Patients were excluded if (1) they did not receive recommended postoperative medications or chemoradiotherapy or (2) if death occurred because of accidents or diseases in other systems not related to the primary carcinoma and surgery.

\section{Treatments}

All patients received standardized radical hepatic metastasis resection using either GA alone or EGA. The anaesthesia type was chosen in overall consideration of the patients' general conditions as well as by the anaesthesiologist's decision. GA was induced as combined intravenous-inhalant anaesthesia, and the choice of hypnotic or sedative anaesthetic drugs, analgesics and muscle relaxants were at the discretion of the attending anaesthesiologists; such medications included propofol, sevoflurane, dexmedetomidine, fentanyl, remifentanil, sufentanil, and atracurium. Pre-operative medication and postoperative analgesia were allowed and accepted. EGA was induced as a standard technique: Patients received epidural catheterization at thoracic 7-10 interspaces before anaesthesia induction. A test dose of $2 \%$ lidocaine was injected. After induction of anaesthesia, a bolus of $7-10 \mathrm{ml} \quad 0.75 \%$ ropivacaine was administered via a thoracicepidural catheterand followed by a continuous epidural infusion of $0.2 \%$ ropivacaine at a rate of $6 \mathrm{ml}$ per hour. We stopped the epidural infusion and removed the epidural catheter at the end of the operation. The mean arterial pressure 
(MAP) was maintained from 55 to $65 \mathrm{mmHg}$ during anaesthesia. All operations and anaesthesia occurred at least five years before we started the current study.

\section{Data Collection}

Data were collected through clinical charts from surgeons' offices and institutional databases, and some survival data were accessed through doctors' follow-up investigations based on registry numbers as necessary and if accessible. Medication or chemotherapy in the regimen after surgery was examined, information on adverse events was recorded, and data on each patient's time of death were collected as well as the cause of death. If no effective postoperative data were available, the subjects were effectively removed from the analysis.

\section{Statistical Analysis}

Data are described using descriptive statistics (mean/standard deviation or median/range for continuous variables; absolute number/frequency distribution for categorical variables). To adjust for confounding between cohorts, we performed a chi-squared test and Student's t-test or WilcoxonMann-Whitney test for categorical variables and continuous variables. Survival was compared using Kaplan-Meier survival curves and log-rank tests. Next, we used a proportional hazards regression model (PROC PHREG) to compare the hazard ratio and $95 \%$ confidence interval (CI) for survival between the two cohorts. The significance level was set at $\mathrm{P}<$ 0.05 . All statistical analyses were performed using SPSS (version 22.0, College Station, TX).

\section{Results}

Comparisons between the EGA $(\mathrm{N}=92)$ and GA $(\mathrm{N}=133)$ groups with respect to baseline factors are shown in Table 1. The patients' basic characteristics in the two groups were largely similar. The median (quartiles) recurrence interval for all patients was 10 $(2.5,23)$ months, and the median (quartiles) survival of patients with CRCLMs was $37(30.5,51.5)$ months. There were no deaths in the first 10 months. Lymphatic and peripheral tissue invasion were comparatively rare, and no nerve or vascular invasion was found. As described in Table 2, perioperative epidural analgesia was associated with survival ( $P$ $=0.039$, log-rank test), with an estimated hazard ratio of $0.737(95 \%$ CI $0.551-0.985)$ in the univariate analysis. The Kaplan-Meier estimates of survival for the GA and EGA groups are provided in Figure 1; the shapes and Cox model analysis of the curves over time suggest that GA might provide better outcomes than EGA $[\mathrm{P}=0.028$, hazard ratio of $0.7328(95 \% \mathrm{CI}$ 0.5433-0.9884)]. Table 3 details the multivariate Cox regression results of relationships between survival after metastatic resection and available covariables. In a multivariable model, we analysed the related factors, including age, anaesthesia technique, primary tumour pathology, Dukes' classification, recurrence interval and peripheral tissue interval (model 1, Table 3). Significant differences in anaesthesia techniques were found $(\mathrm{P}=0.044)$, with an adjusted estimated hazard ratio of 0.741 (95\% CI 0.550, 0.998). Age was also associated with survival, with a $P$ value of 0.046 and hazard ratio of $0.988(0.975,1.001)$ in the univariable analysis, and a P value of 0.044 and hazard ratio of $0.741(0.550,0.998)$ in the multivariable analysis. We also performed a subgroup analysis of patients in different age groups ( $<40$ years old, $\geq 40$ but $<60$ years old, and $\geq 60$ years old). The results suggested that no significant differences existed among all three subgroups. [ $(\mathrm{P}$ value, hazard ratio; $0.3777,1.014$ (0.4001 to 2.568$) ; 0.1657,1$ (0.6921 to 1.445$) ; 0.470$, $1.041(0.6061,1,788)$, Figure 2].

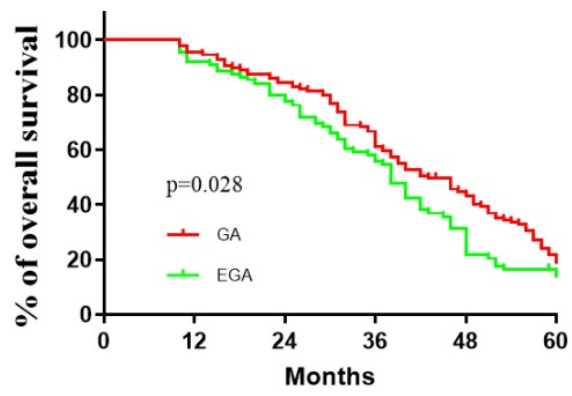

Figure 1. Kaplan-Meier recurrence-free survival estimates for 225 patients given general anaesthesia (GA) and general anaesthesia combined with epidural anesthesia (EGA) during the resection of colorectal carcinoma liver metastases (univariable $\mathrm{P}=0.028$, log-rank test).
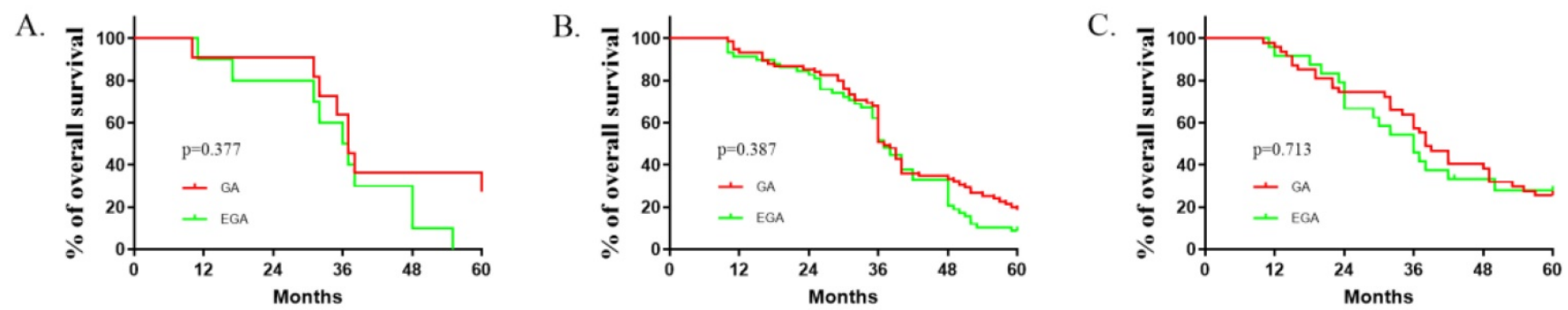

Figure 2. Epidural use and age interaction (age categories). Kaplan-Meier time to survival (A: $<40$ years old; B: $\geq 40$ and $<60$ years old; C: $\geq 60$ years old). The reversal of the relationship of interest for the two age groups suggests an interaction between epidural use and age. 
Table 1. Basic Characteristics

\begin{tabular}{|c|c|c|c|c|}
\hline Factor & Level & GA $(N=133)$ & EGA $(\mathrm{N}=92)$ & P Value ${ }^{*}$ \\
\hline Age (year) & & $54(48,63)$ & $54(47,60)$ & 0.241 \\
\hline Gender & Male & $85(63.9)$ & $60(65.2)$ & $0.840 \dagger$ \\
\hline Ever smoker & Yes & $37(27.8)$ & $31(33.7)$ & $0.377 \dagger$ \\
\hline \multirow[t]{2}{*}{ Primary tumour site } & $\mathrm{R}$ & $26(19.5)$ & $19(20.7)$ & $0.696 \dagger$ \\
\hline & $\mathrm{L}$ & 106(79.7) & $73(79.3)$ & \\
\hline \multirow[t]{4}{*}{ Primary tumour pathology } & $\mathrm{i}$ & $1(0)$ & $2(2.1)$ & 0.272 \\
\hline & ii & $7(5.1)$ & $13(14.1)$ & \\
\hline & iii & $116(87.2)$ & $72(78.3)$ & \\
\hline & iv & $9(6.8)$ & $5(5.4)$ & \\
\hline \multirow[t]{4}{*}{ Dukes' classification } & B & $32(24.1)$ & $30(32.6)$ & 0.064 \\
\hline & $\mathrm{C} 1$ & $16(12.0)$ & $15(16.3)$ & \\
\hline & $\mathrm{C} 2$ & $44(33.1)$ & $26(28.2)$ & \\
\hline & $\mathrm{D}$ & $41(30.8)$ & $21(22.8)$ & \\
\hline Recurrence interval (month) & & $10(2.5,20)$ & $13(2.5,29)$ & 0.279 \\
\hline \multirow[t]{4}{*}{ Number of metastases } & 1 & $75(56.4)$ & $50(54.3)$ & 0.513 \\
\hline & 2 & $26(19.5)$ & $20(21.7)$ & \\
\hline & 3 & $11(8.3)$ & $8(8.7)$ & \\
\hline & $>4$ & $21(15.8)$ & $14(15.2)$ & \\
\hline Diameter of metastases & & $4.5(3,6)$ & $4(2.8,6)$ & 0.646 \\
\hline Lymphatic invasion & Yes & $2(1.5)$ & $2(2.1)$ & 0.709 \\
\hline Peripheral tissue invasion & Yes & $5(3.8)$ & $4(4.3)$ & 0.825 \\
\hline Hypertension or diabetes & Yes & $27(20.3)$ & $15(16.3)$ & 0.370 \\
\hline WBC* $10^{9}$ (pre-operation) & & $5.6(2.1)$ & $5.5(1.6)$ & 0.819 \\
\hline $\mathrm{WBC}^{*} 10^{9}$ (post-operation) & & $12.1(4.1)$ & $13.7(4.2)$ & 0.183 \\
\hline $\begin{array}{l}\text { Percent difference of WBC } \\
\left({ }^{*} 100 \%\right)\end{array}$ & & $1.3(0.8)$ & $1.7(1.2)$ & 0.150 \\
\hline Survival (months) & & $37(29.5,48)$ & $38(31,57.5)$ & 0.218 \\
\hline
\end{tabular}

Table 2. Univariable Associations with Cancer Recurrence: Cox Regression Model Results

\begin{tabular}{lll}
\hline Factor (Reference/Unit Increase) & P Value & Hazard Ratio $(\mathbf{9 5} \% \mathbf{C I})^{*}$ \\
\hline Age(10 year) & 0.046 & $0.988(0.975,1.001)$ \\
Gender (male) & 0.814 & $0.946(0.712,1.305)$ \\
Anaesthesia technique (no epidural) & 0.039 & $0.737(0.551,0.985)$ \\
Ever smoker (yes) & 0.928 & $1.015(0.704,1.390)$ \\
Primary tumour site (R vs. L) & 0.257 & $0.818(0.578,1.158)$ \\
Primary tumour pathology (per unit) & 0.093 & $0.797(0.595,1.069)$ \\
Dukes' classification (per unit) & 0.766 & $1.026(0.864,1.219)$ \\
Number of recurrences (per rank) & 0.722 & $0.975(0.846,1.123)$ \\
Recurrence interval (1 yr) & 0.061 & $1.024(0.934,1.123)$ \\
Diameter of metastases (1 cm) & 0.878 & $0.996(0.948,1.047)$ \\
Lymphatic invasion (yes) & 0.712 & $0.792(0.231,2.726)$ \\
WBC ${ }^{*} 10^{9}$ (pre-operation) & 0.689 & $0.985(0.623,1.479)$ \\
Peripheral tissue invasion (yes) & 0.097 & $0.459(0.211,1.163)$ \\
Hypotension or diabetes (yes) & 0.694 & $1.044(0.843,1.292)$ \\
\hline
\end{tabular}

Risk of cancer recurrence per unit increase in factor. R: right hemicolon, L: left hemicolon and rectum.

Table 3. Multivariable Associations with Cancer Recurrence: Cox Regression Model Results

\begin{tabular}{lll}
\hline Factor (Reference/Unit Increase) & P Value & Hazard Ratio (95\% CI) \\
\hline Age (10yr) & 0.048 & $0.988(0.975,1.001)$ \\
Anaesthesia technique (no epidural) & 0.044 & $0.741(0.550,0.998)$ \\
Primary tumour pathology (per unit) & 0.223 & $0.839(0.633,1.113)$ \\
Recurrence interval (1 yr) & 0.052 & $1.016(0.931,1.312)$ \\
Peripheral tissue invasion (yes) & 0.165 & $0.992(0.647,1.312)$ \\
\hline
\end{tabular}

Risk of cancer recurrence per unit increase in factor. R: right hemicolon, L: left hemicolon and rectum.

\section{Discussion}

In a retrospective analysis of patients undergoing surgical treatments for breast cancer and prostate cancer, Gottschalk et al. and Biki et al. reported that EGA was associated with a longer recurrence-free survival.[20,21] Similar results were demonstrated in patients with melanoma and prostate cancer, for which EGA was associated with a lower risk of recurrence.[22,23] However, some other studies reported contrasting results showing that EGA cannot provide better outcomes in cancer patients.[21,24-26] A meta-analysis on this topic showed that EGA might be associated with an improvement in the prognosis of patients with operable prostate cancer, and no significant improvement in colorectal cancer was found.[18] These studies suggest that the potential benefits of EGA may depend on the tumour type, which may be related to the different pathologies and metastases. Thus, large-scale clinical studies involving different cancer types are needed to investigate the potential influence of the anaesthetic technique used during surgery on cancer-related outcomes.

This study was based on a chart review of medical records, with a sample size of 225 , and reflected the survival of patients following CRCLM resection in a real-world population in China. Our results suggested that EGA use seems to provide no benefit with respect to the rate of overall survival in CRCLM patients. For GA patients, the survival seems slightly longer than that of EGA patients. In contrast with the results obtained by Gottschalk et al. showing that EGA might improve the outcomes of elderly individuals undergoing colorectal cancer surgery, it seemed that patients older than 60 years in our study did not benefit from EGA treatment. It is possible that EGA might influence the cancer-related outcomes of patients through different mechanisms and that only a subgroup of patients with certain constraints may benefit from it.

Previous studies concluded that the possible mechanism of EGA in cancer-related survival might be complicated and comprehensive, including haemodynamic factors and the stress and immune responses, as epidural use during surgery could decrease the need for opioid use, preventing pain and decreasing the activation of inflammatory factors, thus promoting tumour surveillance.[27] The elevated NK cell activity of the immune system induced by lidocaine epidural infusion also greatly benefits elderly patients undergoing colorectal cancer surgery. However, our study provides a diverse outcome showing that EGA probably does not perform well or may even be worse than GA treatment with respect to the survival of post-surgical CRCLM patients. 
Possible causes may include the following: First, EGA is an invasive operation; for patients with advanced cancer, such as CRCLMs, the trauma from epidural treatment may be greater than its potential immune benefits. Second, the benefits of EGA are limited to specific tumour types and special populations and thus are not universally applicable. Third, although the baseline characteristics of the two different groups were compared and deemed largely similar, anaesthesiologists may still choose to use a combined epidural technique for patients with special situations or high risks for haemodynamic stability intraoperatively and better analgesia postoperatively. Considering this fact, it is more appropriate to conclude that EGA use does not provide obvious long-term survival benefits for CRCLM patients treated by resection.

Our choice of primary statistical analysis was a multivariable model to adjust for confounding variables, enabling the assessment of the effect of each potential confounding variable on the outcome simultaneously and the effect of variables interacting with EGA. A sensitivity analysis using propensity score analysis yielded similar results and the same conclusions.

This analysis has several limitations or factors that are unavoidable. First, epidural use in GA has waned due to the progression of GA techniques and the potential hazards of invasive techniques. Patients using EGA are often under special conditions or have high risks. Second, we could not determine whether the epidural use in each subject was sufficient or up to standard. Similarly, we could not determine whether other surgical or anaesthesia techniques were performed using standard criteria. More importantly, unrecorded patient characteristics that influenced the risk of mortality may have influenced the anaesthetic management. Since surgical outcomes may be affected by multiple factors and tumour types, conclusions on the benefits of EGA should be drawn with caution, as certain anaesthesia techniques may not have a remarkable effect on the postoperative survival of cancer patients. More prospective studies, including randomized controlled trials and retrospective studies with a large scale, are needed. In addition, since we compared only the outcomes of general survival between patients with the two anaesthesia techniques, more studies are needed to further investigate other short-term or long-term outcomes, such as blood transfusion and ICU length of stay, to provide a better reference for clinicians.

\section{Acknowledgements}

The authors thank Ke-Hong Zhang, MD, PhD (IVY Medical Editing) for some suggestions for the article revise.

\section{Funding}

This study was supported by a grant from the National Natural Science Foundation of China: (NSFC, No.81371511) and Shanghai Municipal Commission of Health and Family Planning Funding for Key Developing Disciplines (2015ZB0101).

\section{Competing Interests}

The authors have declared that no competing interest exists.

\section{References}

1. Siegel R, Ma J, Zou Z, et al. Cancer statistics, 2014. CA Cancer J Clin. 2014; 64: 9-29.

2. Leporrier J, Maurel J, Chiche L, et al. A population-based study of the incidence, management and prognosis of hepatic metastases from colorectal cancer. British Journal of Surgery. 2006; 93: 465-474.

3. Adam R. Chemotherapy and surgery: new perspectives on the treatment of unresectable liver metastases. Annals of Oncology. 2003; 14: ii13-6.

4. Shimada H, Tanaka K, Endou I, et al. Treatment of colorectal liver metastases: a review. Langenbecks Arch Surg. 2009; 4: 56-62.

5. Adam R, Haller DG, Poston G, et al. Toward optimized front-line therapeutic strategies in patients with metastatic colorectal cancer-an expert review from the International Congress on Anti-Cancer Treatment (ICACT) 2009. Annals of Oncology Official Journal of the European Society for Medical Oncology. 2010; 21: 1579-84.

6. Mannoor K, Shen J, Liao J, et al. Small nucleolar RNA signatures of lung tumor-initiating cells. Molecular Cancer. 2014; 13: 104-104

7. Ito $\mathrm{K}$, Govindarajan $\mathrm{A}$, Ito $\mathrm{H}$, et al. Surgical treatment of hepatic colorectal metastasis: evolving role in the setting of improving systemic therapies and ablative treatments in the 21st century. Cancer Journal. 2010; 34: 103-110.

8. Koppe MJ, Boerman OC, Oyen WJ, et al. Peritoneal carcinomatosis of colorectal origin: incidence and current treatment strategies. Annals of Surgery. 2006; 243: 212-22.

9. Kato T, Yasui K, Hirai T, et al. Therapeutic results for hepatic metastasis of colorectal cancer with special reference to effectiveness of hepatectomy-Analysis of prognostic factors for 763 cases recorded at 18 institutions. Diseases of the Colon \& Rectum. 2003; 46: 22-31.

10. Spelt L, Andersson B, Nilsson J, et al. Prognostic models for outcome following liver resection for colorectal cancer metastases: A systematic review. European Journal of Surgical Oncology. 2012; 38: 16-24.

11. Snyder GL, Greenberg S. Effect of anaesthetic technique and other perioperative factors on cancer recurrence. British Journal of Anaesthesia. 2010; 105: 106-115.

12. Casati L, Fernández-Galinski S, Barrera E, et al. Isoflurane requirements during combined general/epidural anesthesia for major abdominal surgery. Anesthesia \& Analgesia. 2002; 94: 1331-7.

13. Kaneda K, Takeuchi J, Yakushiji T, et al. [A case of anesthesia for a patient with a huge pheochromocytoma accompanying difficulty in hemodynamics control]. Masui the Japanese Journal of Anesthesiology 2006; 55: 900-3.

14. Sotunmbi PT, Shittu OB, Windokun A, et al. Combined general and epidural anaesthesia for excision of phaeochromocytoma--a unique and safe technique. African Journal of Medicine \& Medical Sciences. 2000; 29: 319-22.

15. Hiller JG, Hacking MB, Link EK, et al. Perioperative epidural analgesia reduces cancer recurrence after gastro-oesophageal surgery. Acta Anaesthesiologica Scandinavica. 2014; 58: 281-290.

16. Merquiol F, Montelimard AS, Nourissat A, et al. Cervical epidural anesthesia is associated with increased cancer-free survival in laryngeal and hypopharyngeal cancer surgery: a retrospective propensity-matched analysis. Reg Anesth Pain Med. 2013; 38: 398-402.

17. Bar-Yosef S, Melamed R, Page GG, et al. Attenuation of the tumor-promoting effect of surgery by spinal blockade in rats. Anesthesiology. 2001; 94: 1066-73.

18. Pei L, Gang T, Lei W, et al. Comparison of Combined General-Epidural Anesthesia with General Anesthesia Effects on Survival and Cancer Recurrence: A Meta-Analysis of Retrospective and Prospective Studies. Plos One 2014; 9: e114667.

19. Viganò L, Procopio F, Cimino M, et al. Is Tumor Detachment from Vascular Structures Equivalent to R0 Resection in Surgery for Colorectal Liver Metastases? An Observational Cohort. Ann. Surg. Oncol. 2016; 23: 1352-60.

20. Biki B, Mascha E, Moriarty DC, et al. Anesthetic technique for radical prostatectomy surgery affects cancer recurrence: a retrospective analysis. Anesthesiology. 2008; 109: 180-187.

21. Gottschalk A, Ford JG, Regelin CC, et al. Association between Epidural Analgesia and Cancer Recurrence after Colorectal Cancer Surgery. Anesthesiology. 2010; 113: 27-34.

22. Exadaktylos AK, Buggy DJ, Moriarty DC, et al. Can anesthetic technique for primary breast cancer surgery affect recurrence or metastasis? Anesthesiology. 2006; 105: 660-664. 
23. Wuethrich PY, Hsu Schmitz SF, Kessler TM, et al. Potential influence of the anesthetic technique used during open radical prostatectomy on prostate cancer-related outcome: a retrospective study. Anesthesiology. 2010; 113: 570-6.

24. Binczak M, Tournay E, Billard V, et al. Major abdominal surgery for cancer: does epidural analgesia have a long-term effect on recurrence-free and overall survival? Ann Fr Anesth Reanim. 2013; 32: e81-8.

25. Lacassie HJ, Cartagena J, Brañes J, et al. The relationship between neuraxial anesthesia and advanced ovarian cancer-related outcomes in the Chilean population. Anesthesia \& Analgesia. 2013; 117: 653-660.

26. Myles PS, Peyton P, Silbert B, et al. Perioperative epidural analgesia for major abdominal surgery for cancer and recurrence-free survival: randomised trial. BMJ. 2011; 342: 746-746.

27. Cummings Kenneth C, Xu F, Cummings Linda C, et al. A Comparison of Epidural Analgesia and Traditional Pain Management Effects on Survival and Cancer Recurrence after Colectomy: a Population-based Study. Anesthesiology. 2012; 116: 797-806. 